\title{
CARACTERÍSTICAS CLÍNICAS Y EPIDEMIOLÓGICAS DE LOS PACIENTES HOSPITALIZADOS POR INFLUENZA A (H1N1) EN TUXTLA GUTIÉRREZ; CHIAPAS, MÉXICO
}

\section{CLINICAL AND EPIDEMIOLOGICAL CHARACTERISTICS OF PATIENTS HOSPITALIZED FOR INFLUENZA A (H1N1) IN TUXTLA GUTIÉRREZ; CHIAPAS, MEXICO}

\author{
José Antonio Sánchez Lozano $•$ antonioslozano@gmail.com \\ Rosa Margarita Durán García • rosa.duran@unicach.mx \\ María Georgina Rivas Bocanegraํ • georgina.rivas@unicach.mx \\ Ángel Gutiérrez Zavala • guzava@hotmail.com \\ Oscar Alfaro Macías² • oalfaro22@hotmail.com
}

1 Universidad de Ciencias y Artes de Chiapas, México

2 Laboratorio Estatal de Salud Pública del Instituto de Salud del estado de Chiapas, México 
Para citar este artículo:

Sánchez Lozano, J. A., Durán García, R. M., Rivas Bocanegra, M. G., Gutiérrez Zavala, Ángel, \& Alfaro Macías, O. (2020). Características clínicas y epidemiológicas de los pacientes hospitalizados por influenza A (H1N1) en Tuxtla Gutiérrez; Chiapas, México. ESPACIO I+D, INNOVACIÓN MÁS DESARROLLO, 9(24). https://doi.org/10.31644/IMASD.24.2020.a04

\section{RESUMEN}

Con el fin de evaluar las características clínicas y epidemiológicas de los pacientes hospitalizados por influenza A H1N1 en Tuxtla Gutiérrez, Chiapas, México, se realizó un estudio transversal y serie de casos. Se encontró que un total de 6.120 muestras de personas sospechosas de estar infectadas por el virus de la gripe H1N1; 51.5\% $(3,151)$ fueron positivos, con el mayor porcentaje de casos en la Ciudad de Tuxtla Gutiérrez. De los 1,567 casos positivos (49.7\%), eran mujeres. La edad promedio de la población estudiada fue de $21.5 \pm 4.5$ años. El grupo más afectado fue de 30 a 59 años (48.7\%), seguido por el grupo de 15 a 29 años (29.5\%). Las amas de casa y los estudiantes (madre e hijo), fueron un grupo vulnerable a enfermarse; porque presentaron los casos más altos (62\%); en cuanto a la clasificación de riesgo, la patología concomitante más frecuente fue diabetes mellitus en 11.5\% $(n=9)$ seguida de hipertensión arterial con $8 \%(n=6)$ y diferentes enfermedades respiratorias 10.2\% $(n=8)$. Asimismo, se encontró que la tos y el malestar general estaban presentes en la mayoría de los pacientes.

\section{Palabras clave}

Influenza A ( $\mathrm{H} 1 \mathrm{~N} 1)$, características epidemiológicas, Chiapas. 
In order to evaluate the clinical and epidemiological characteristics of patients hospitalized for influenza A H1N1 in Tuxtla Gutiérrez, Chiapas, Mexico, a cross-sectional study and case series were conducted. It was found that a total of 6,120 samples of people suspected of being infected by the H1N1 influenza virus; $51.5 \%(3,151))$ were positive, with the highest percentage of cases in the City of Tuxtla Gutiérrez. Of the 1,567 positive cases $(49.7 \%)$, they were women. The average age of the population studied was $21.5 \pm 4.5$ years. The most affected group was 30 to 50 years $(48.7 \%)$, followed by the group of 15 to 29 years (29.5\%). Housewives and students (mother and son), were a vulnerable group to get sick; because they presented the highest cases (62\%); regarding the risk classification, the most frequent concomitant pathology was diabetes mellitus for $11.5 \%(\mathrm{n}=9)$ followed by arterial hypertension with $8 \%(\mathrm{n}=6)$ and different respiratory diseases $10.2 \%(n=8)$, Likewise, it was found that cough and general malaise were present in the majority of patients.

\section{Keywords}

Influenza A (H1N1), epidemiological characteristics, Chiapas. 
L a influenza es una enfermedad transmisible respiratoria que aparece de manera novedosa en las poblaciones humanas, con una rápido incremento en su incidencia y en su expansión geográfica. Dicha emergencia es posible cuando, a lo largo del tiempo, la infección en animales traspasa las barreras de especie debido a la transformación del genoma viral, infectando a los seres humanos (Mc Closkey, Osman, Alimuddin \& Heymann, 2014).

De los cuatro tipos de virus de la influenza A, B, C y D, los tipos A y $B$ causan epidemias estacionales cada año, generalmente en los meses de invierno; los otros dos no suelen causar infecciones en humanos o son muy leves (CDC, 2019). Los virus tipo A tienen como reservorios a diferentes especies de aves e infectan a puercos, caballos y otros mamíferos, así como a los humanos; mutan continua y rápidamente y exhiben un alto grado de patogenicidad y virulencia (Kumar, Asha, kahnna, Ronsard, Meseko, Sanikas, 2017). Los virus tipo A son los únicos que pueden desencadenar pandemias de influenza humana, clasificándose en subtipos a partir de dos proteínas de superficie: la hemaglutinina y neuraminidasa, las cuales le dan su capacidad de infectividad y patogencidad, identificándose 18 subtipos de la primera y 11 de la segunda, con múltiples combinaciones, de las cuales 131 han sido detectadas en la naturaleza (CDC, 2019).

El virus A (H1N1) pdmo9 por su nombre científico, que surgiera en la primavera del 2009 y que causará la pandemia, se encuentra desde entonces en circulación estacional en todo el mundo; con cambios genéticos prácticamente mínimos en su antigenicidad (CDC, 2010). Actualmente están en circulación en las poblaciones humanas los tipos A (H1N1) pdmo9 y A ( H3 N2), éste último con cambios más rápidos genética y antigénicamente (CDC, 2019), el cual representó la mayoría de los casos en todo el mundo (ops, 2019). En México desde el 2009 hay un predominio de los mismos subtipos virales, predominando a veces el A (H3 N2) como en la temporada de influenza 2017-2018, o a veces el A (H1N1) pdmo9 en la reciente temporada del 2018-2019 (Secretaría de salud 2019, 5).

Según el reporte de la OMs, 18500 muertes fueron confirmadas por laboratorio e nivel mundial, entre abril 2009 a agosto 2010. Dicha cifra representaría una pequeña fracción de las muertes que muy probablemente ocurrieron asociadas a influenza, pues dado que el total de la media de las estimaciones de las muertes debidas a A (H1N1) pdmog por país, habría estado en un rango de 105, 700 a 395, 600; que al incluir las muertes claramente asociadas a comorbilidad cardiovascular o respiratoria se elevaría de 151, 700 a 575, 400; la mayoría en población adulta joven y madura, en el rango de 18 a 64 años. Más del 51\% de las muertes habrían ocurrido en África y Sudeste Asiático; en América y el Pacífico Occidental la cifra de muertes asociadas a influenza A (H1N1) sería 6 veces más alta en poblaciones indí- 
genas que en las no indígenas. En general entre el $0.001 \%$ al $0.007 \%$ de la población mundial habría muerto durante el primer año de la circulación del virus entre 2009 y 2010 , que se elevaría a 0.001 a $0.011 \%$ al agregar la comorbilidad asociada a las muertes por influenza; menos o similar que lo registrado en otras pandemias, excepto la de 1918 (Dawood, et al, 2012).

En México, entre abril y mayo del 2009, se realizó un análisis preliminar entre casos de muerte por influenza, por parte de la Secretaría de Salud. La epidemia que comenzó oficialmente en marzo en el centro del país, tuvo un comportamiento bifásico en su curva de morbilidad por el desplazamiento de la misma hacia los estados del sureste, donde se ubica Chiapas. Si bien el registro de las defunciones confirmadas por laboratorio comenzó en abril, es posible que anteriormente hubiera otras no registradas, en la medida que no se sabía que se estaba frente a una epidemia. El 79\% de la mortalidad se concentró en los menores de 30 años. La media de la tasa de mortalidad fue de 1.1 casos por millón de habitantes, sin embargo la tasa fue superior a la media entre los 20 y 59 años, con 1.5 y 1.9 por millón, respectivamente. La letalidad fue del $2.2 \%$ en el período citado. Solo el $17 \%$ de los 122 casos estudiados recibió atención hospitalaria las primeras 72 horas y hasta $42 \%$ murió dentro de ese lapso, es decir que el resto de los casos tuvo un retraso en la oportunidad de la atención además de la calidad de la misma al inicio de la epidemia. El 58 \% de los fallecidos tenía alguna comorbilidad (FajardoDolci, et al, 2009).

En Chiapas, la epidemia de influenza comenzó de hecho a finales de junio del 2009 y en el mes de julio los enfermos graves se concentraron en el hospital regional de la ciudad capital Tuxtla Gutiérrez, que para atender la severidad del padecimiento solo contaba con 6 camas de terapia intensiva y algunas de cuidados intermedios. No se encontró información publicada referente al comportamiento de la morbilidad y mortalidad de la epidemia en el estado, la que existe, se refiere justamente a las carencias y necesidades en instalaciones, equipo e insumos así como de personal adiestrado y de procedimientos estandarizados para hacer frente a la situación, que la federación trató de remediar con la adquisición de equipo y capacitación de personal local. Quizás lo más grave fue la aparente insuficiencia en la organización del sistema de salud por parte de las autoridades estatales, que no dimensionaron el riesgo que presentaba la epidemia para la población chiapaneca (Volkow et al, 2011).

El estudio que se presenta a continuación tiene como objetivo llenar un vacío de informaciónón, al describir clínica y epidemiológicamente el comportamiento de la epidemia en el estado de Chiapas en el 2009, a partir de los casos hospitalizados y confirmados por influenza A (H1N1) pdmog, en el hospital regional "Dr. Rafael Pascacio Gamboa” del Instituto de Salud de Tuxtla Gutiérrez, Chiapas. 


\section{MATERIAL Y MÉTODO}

La población objeto de estudio estuvo constituida por todos los pacientes, de cualquier edad y ambos sexos, que ingresaron al hospital general regional, en la ciudad de Tuxtla Gutiérrez, Chiapas; con el diagnóstico confirmado de influenza por el virus A H1N1, mediante la prueba transcripción inversa de la reacción en cadena de la polimerasa (RT-PCR), del 24 de abril de 2009 al 21 de septiembre del mismo año.

El tipo de estudio fue de serie de casos. Los datos fueron recolectados de los expedientes clínicos de pacientes hospitalizados y se complementó la información con los estudios de caso, para notificación epidemiológica, con el formato del Sistema Único de Información para la Vigilancia Epidemiológica (suive), donde se confirmó la realización de la toma de muestra, con el método para la detección del virus A (H1N1).

Las variables de interés fueron: sociodemográficas, clínicas y los antecedentes personales patológicas y no patológicas, así como aspectos relacionados con el tratamiento. Una vez recolectada la información, se elaboró una base de datos en el programa estadístico SPSS, versión 18. Posteriormente se realizó un análisis descriptivo univariado, seguido de un análisis bivariado.

Los criterios de inclusión fueron: haber ingresado al Hospital General Regional de Tuxtla Gutiérrez, contar con estudio epidemiológico de caso y tener el diagnóstico confirmado de influenza A (H1N1), por medio de la prueba de RT-PCR realizada en el Laboratorio Estatal de Salud Pública; y los de exclusión: el ser paciente ambulatorio, tener otro tipo de examen confirmatorio de la enfermedad que no fuese RT-PCR, pacientes que no tengan expediente clínico.

\section{RESULTADOS}

Durante el periodo de estudio se hospitalizaron 217 pacientes, de los cuales 89 ( $41 \%)$ resultaron positivos a influenza A H1N1 a través de la RT-PCR; entre estos 78 (87.6\%) cumplieron con los criterios de inclusión. En la tabla 1 se observa que el grupo de 30 a 59 fue el más afectado, seguido por el de 15 a 19 años. 


\section{Tabla 1}

Frecuencia de la enfermedad según el grupo de edad entre los pacientes hospitalizados del estudio, en el estado de Chiapas, México; del 24 abril al 21 septiembre 2009

\begin{tabular}{ccc}
\hline Grupo de edad & N & $\%$ \\
14 años y menos & 1 & 16. \\
15 a 29 años & 2 & 29. \\
30 a 59 años & 3 & 48. \\
60 años y más & 4 & 5. \\
Total & 7 & 10 \\
\hline
\end{tabular}

Fuente: expediente clínico del Hospital General Regional “Dr. Rafael Pascacio Gamboa” de Tuxtla Gutiérrez, Chiapas, del Instituto de Salud

Las mujeres conformaron la mayoría de los pacientes hospitalizados con el 60.3\%, ( $n=47)$, que se ocupaban generalmente como amas de casa. Las afectadas en edad reproductiva representaron el $72.3 \%(n=34)$ y seis de ellas (12.8\%) cursaban con un estado obstétrico (cuatro con embarazo y dos en puerperio), la edad promedio de las mujeres fue de 21.5 años.

Los hombres constituyeron el $39.7 \%$ de los pacientes $(n=31)$; la edad promedio fue de 32 años (rango 3 a 70), y sus actividades más importantes fueron la de estudiante, 12 (38.7\%) y la de chofer, 5 (16\%).

De toda la serie, se destacó que en los mayores de 15 años, cuatro (5\%) eran analfabetas, ocho (11.5\%) únicamente tenían primaria y 10 (12.8\%) tenían secundaria; sin embargo en 22 pacientes (28.2\%) no se registró la escolaridad en el expediente.

Con respecto a su lugar de residencia, el $82 \%(n=64)$ pertenecían a los municipios que integran la Jurisdicción Sanitaria (J.S) No 1cuya sede es Tuxtla Gutiérrez, de los cuales 46 (71.8\%) eran residentes de la ciudad capital. El 15.4\% $(n=12)$ fueron referidos de otras jurisdicciones. Finalmente dos pacientes (2.6\%) se identificaron como turistas, procedentes de; Coatzacoalcos (estado de Veracruz) y Villahermosa (estado de Tabasco).

Con relación a la clasificación de riesgos el 11.5\% tenían diabetes mellitus la patología concomitante más frecuente, seguido de la hipertensión arterial con $8 \%(n=6)$ y diferentes enfermedades respiratorias $10.2 \%(n=8)$, entre las que destacaron el asma con 5.1\% $(n=4)$; además de otras como tuberculosis pulmonar, EPOC, rinitis crónica, con un caso cada una. Al revisar los antecedentes personales no patológicos, la convivencia con animales $28.3 \%$ y el hacinamiento $24.4 \%$ resultaron ser las condiciones más frecuentes.

Como se observa en tabla 2 , 23 pacientes presentaron riesgos patológicos que representaron el $30 \%$ y cerca del $63 \%$ no patológicos, mientras 27 pacientes tenían de 3 o más riesgos. 
Tabla 2

Distribución de pacientes según tipo y número de riesgos

\begin{tabular}{ccc}
\hline Antecedentes & $\mathbf{n}$ & $\%$ \\
De riesgo patológico & 23 & 29.5 \\
De riesgo no patológico & 49 & 62.8 \\
Pacientes con máximo dos riesgos & 26 & 33.3 \\
Pacientes entre 3 y 6 riesgos & 23 & 29.5 \\
Pacientes con Siete y más riesgos & 4 & 5.0 \\
\hline
\end{tabular}

Fuente: expediente clínico del Hospital General Regional “Dr. Rafael Pascacio Gamboa” de Tuxtla Gutiérrez, Chiapas, del Instituto de Salud

Con respecto al tiempo medio entre el inicio de los síntomas y el momento en que se presentaron los pacientes a solicitar atención médica, fue de 3.7 días, con un rango de o hasta 14 días. De los pacientes hospitalizados, 47 (60.3\%), estaban igual o por debajo de la media; la moda fue de 1 día en 25 pacientes (32\%). El promedio de días de hospitalización fue de cuatro (rango 1 a 24 ).

Al momento de la hospitalización se realizó una historia clínica enfocada a identificar el cuadro clínico inicial, con la finalidad de integrar un diagnóstico sindromático o nosológico y se encontró que la tos y el malestar general, estuvieron presentes en la mayoría de los pacientes (tabla 3).

\section{Tabla 3}

Distribución porcentual de signos y síntomas de los casos al ingreso

\begin{tabular}{ccc}
\hline Signos y Síntomas & $\mathbf{n}$ & $\%$ \\
Tos & 71 & 91.0 \\
Ataque al estado general & 63 & 80.8 \\
Disnea & 48 & 61.5 \\
Fiebre & 43 & 55.0 \\
Cuadro gripal & 27 & 34.6 \\
Cefalea & 24 & 30.8 \\
Dolor y ardor de garganta & 14 & 18.0 \\
Dolor torácico & 8 & 10.3 \\
Cianosis & 5 & 6.4 \\
\hline
\end{tabular}

Fuente: expediente clínico del Hospital General Regional “Dr. Rafael Pascacio Gamboa” de Tuxtla Gutiérrez, Chiapas, del Instituto de Salud

La tos tuvo una duración media de 3.4 días (moda de un día en 28 pacientes; rango $1 \mathrm{a} 14)$. Los pacientes que tuvieron disnea fueron 48 (61.5\%), con una media de 2.3 días. Finalmente la cianosis se registró en cinco pacientes (6\%); todos con un día de evolución. 
De acuerdo a los criterios de "American College of Chest Physcians Society for Critical Care Medicine Consensus Conference", se clasificaron a 40 pacientes ( $51.3 \%)$ con síndrome de inflamación sistémica, de los cuales $35(87.5 \%)$, tenían al menos una enfermedad o antecedente considerado de riesgo; incluyendo a cuatro pacientes que tenían antecedentes de enfermedades respiratorias crónicas.

Excluyendo a los que fallecieron; 10 pacientes (25\%) tuvieron sepsis como primer causa de complicaciones y cinco de ellos (12.5\%), cursaron con choque séptico, que finalmente condicionó que dos $(5 \%)$ tuvieran insuficiencia renal aguda. En este grupo de pacientes, aumentó el promedio a cinco días (rango o a 14), para solicitar atención, desde el inicio de los síntomas.

El criterio de ingreso a la Unidad de Cuidados Intensivos (UCI), fue la insuficiencia respiratoria. De los 40 pacientes con síndrome de inflamación sistémica, 20 (50\%), ingresaron a la UCI, cuya edad promedio fue de 35 años (rango 21 a 52). El promedio de días de estancia en la UCI, fue de 10 (rango 6 a 19).

Con relación al promedio de días en que estos pacientes solicitaron atención médica desde el inicio de la enfermedad fue de seis (rango 2 a 14). De los cuales nueve pacientes (45\%), se encontraban igual o por encima de esta media.

Para todos los hospitalizados, el promedio de días, entre el inicio de los síntomas y la toma de muestra con hisopo para la RT-PCR, fue de 4.3 días (rango 1 a 15); el 63\% ( $n=49)$, se encontraba igual o por debajo de esta media; la moda fue de un día para 22 pacientes (28\%).

Con relación al tiempo de inicio de la terapia antiviral, 33 pacientes (44\%), recibieron oseltamivir dentro de las primeras 48 horas del inicio de los síntomas; sin registro de fallecimientos durante este periodo. El primer fallecimiento se registró al tercer día de inicio de los síntomas. El promedio de tiempo, desde la aparición de los signos, hasta el inicio del tratamiento antiviral fue de 4.3 días (rango, o a 16), y en 29 pacientes (38.7\%), se inició después de esta media. Se complementó el manejo con el uso de antimicrobianos en 20 pacientes $(25.6 \%)$.

El número de defunciones fue de 15 pacientes (19.2\%); 10 fueron mujeres (66.7\%), entre ellas una embarazada y una puérpera, así como 5 hombres $(33.3 \%)$. El promedio de edad de quienes fallecieron fue de 35 años (mujeres 32 años y hombres 41 años). El rango de edad de estos pacientes varió de 23 a 52 años.

Acerca de la escolaridad, 10 de las personas fallecidas contaban con educación básica (66.7\%). Hubo 2 profesionistas. También murió una de las 4 personas analfabetas y en 2 casos no se encontró registro. Al analizar la ocupación, 9 mujeres se dedicaban al hogar y una era maestra. De los hombres, 3 se dedicaban a la construcción, uno de ellos era arquitecto y los otros 2 eran chóferes. 
Por su ubicación geográfica: 11 pacientes (73.3\%), pertenecían al área de influencia de la Jurisdicción Sanitaria (J.S.). No. 1 (Tuxtla Gutiérrez), dos casos a la J.S. No. 2 (San Cristóbal de las Casas), un caso a la Jurisdicción Sanitaria. J.S. No. 8, (Tonalá), y uno de ellos fue turista con residencia en Villahermosa, Tabasco. Por municipio, cinco pacientes tenían su residencia en Tuxtla Gutiérrez, y tres en Jiquipilas, Chiapas.

Con respecto a los antecedentes patológicos de riesgo, el más frecuente fue la diabetes mellitus, con cinco casos (33.3\%), seguido por la obesidad con tres casos (20\%), y de los antecedentes de enfermedades respiratorias crónicas, el asma fue la única presente con un solo caso.

De los antecedentes no patológicos de riesgo; el más frecuente fue la exposición a animales en siete casos (46.7\%), seguida la exposición al humo de leña en cuatro casos (26.7\%), el hacinamiento y la migración estuvieron presentes en tres casos (20\%). El alcoholismo y tabaquismo, solo estuvo presente en un caso para ambos; sin embargo en 5 casos, para cada antecedente, no se encontró registro. En resumen, once pacientes, tuvieron 3 factores de riesgo y en un paciente se encontraron 7 .

La tos se caracterizó por ser purulenta y hemoptoica en la mitad de los fallecidos, 49.9\% $(\mathrm{n}=7)$. La disnea también estuvo presente en 14 pacientes (93\%), clasificados entre "grave o muy grave" según la "American Thoracic Society", con una media de tres días (rango 1 a 5 ). Con cianosis cursaron cinco pacientes (33.3\%), todos con un día de evolución.

Las complicaciones que los llevaron a la muerte fueron: la sepsis en 14 de los casos (93.3\%); 12 evolucionaron a choque séptico e insuficiencia renal aguda $(85.7 \%)$.

La estancia promedio de los 15 fallecidos, que además ingresaron a la UCI, fue de nueve días (rango o a 23). La media desde el inicio de los síntomas hasta solicitar la atención médica, fue de seis días (rango 3 a 14); y el promedio desde el inicio de los síntomas a la defunción fue de 16 días (rango 4 a 26).

Cabe hacer mención, que la proporción de fallecimientos fue significativamente mayor en las personas que iniciaron el tratamiento después del cuarto día, 12 (80\%) que en aquellos que lo iniciaron antes, 3 (20\%). La letalidad que se registró durante el periodo de estudio, fue de 4.76/1000 casos confirmados de influenza A (H1N1).

\section{DISCUSIÓN}

En México, la Secretaría de Salud reportó a la oms; entre marzo y abril de 2009, la ocurrencia de tres brotes: en el entonces Distrito Federal y ahora Ciudad de México (854 casos de neumonía, con 59 fallecidos), en San Luis Potosí (24 casos con tres defunciones) y Mexicali (cuatro casos y ninguna 
defunción). El estado de Chiapas comenzó a figurar en las estadísticas hasta el 6 de mayo de 2009, en un informe preliminar de la Secretaria de Salud Federal, que publicaba el primer caso de fallecimiento (Secretaría de Salud 2009).

Así entonces para el 26 de septiembre del 2009, (periodo de tiempo que incluyó el presente estudio), Chiapas ya acumulaba el 12\% de los casos registrados en el ámbito nacional (InDRE; $\mathrm{n}=3561$ ); y a partir del mes de octubre hubo una disminución considerable en el número de casos reportados, existiendo una diferencia para mediados de julio de 2010 , de tan solo 157 positivos en un periodo de 10 meses (InDRE; $n=3718$ ), que en la estadística, llevó al estado del primer lugar de casos confirmados al sexto (Salud México, 2010).

En el estado se observó que el grupo de edad más afectado fue el de 5 a 29 años (65\%), y el promedio de edad cercano a los 31 años; resultado similar a los registrados en la ciudad de México (López, Solís AM, López V, García Rivas D y Lozano JJ,2010) en la estadística nacional (Fajardo et al, 2009) y en otros países tales como: Brasil (Duarte PAD 2009), Canadá (Kumar A et al, 2009), Irán (Mehdi, G M et al, 2009) y China (The National Influenza A Pandemic (H1N1) 2009).

Los grupos de edad considerados de riesgo para enfermar de IRA $(<5 \mathrm{y}$ $>65$ años), tuvieron una tasa general de 5 y $1.5 / 10,000$ respectivamente y la tasa de hospitalización fue de 4/100,000; cifras parecidas a las de otros estudios (Organización Panamericana de la Salud [ops], 2009), (Ayora, TG 1999), (Virus Investigation Team, 2009), (Libster et al, 2010), (Louie JK et al, 2009) (Telo Velosa CS, 2009) y (O’Riordan y Sean, 2009). Cabe señalar que la tasa de ataque general, en la región de Tuxtla Gutiérrez fue de 40/10,00o habitantes, y la de hospitalización fue de 4.5/100 infectados.

Las amas de casa y los estudiantes (madre e hijo), fueron un grupo vulnerable para enfermar, en ellos se presentaron los mayores casos (62\%); sin embargo llama la atención que las amas de casa, en su mayoría, se registraron durante el mes de julio $(n=14)$ con una media el día 4 de julio; y con los datos obtenidos no se constató la relación de madre-estudiante, debido a que el grupo de estudiantes se registró en la mayoría de los casos durante el mes de junio $(n=19)$ con una media el día 7 junio. Tampoco se identificaron grupos familiares completos. Lo anterior permite suponer que el mecanismo de transmisión es el común; de persona a persona; y que siguió un patrón similar al propuesto por Cauchemez S. y colaboradores (2009); cuyo índice afirma que la transmisión en hogares donde viven dos personas es del $29 \%$, y en viviendas donde viven seis personas es del $9 \%$.

En lo que respecta a los antecedentes clínicos patológicos y no patológicos, la obesidad y diabetes mellitus fueron las enfermedades concomitantes más significativas en la población afectada Louie JK et al (2009), Jain S. et al (2009), Pérez Padilla et al (2010), aunque existe un sesgo muy importante 
toda vez que no se registró el $25 \%$ de este dato en el expediente. Algo importante que destacar es que no concuerda con otros estudios que muestran hasta un 60\% de incidencia de estas enfermedades (Louie, JK et al, 2009) (Pérez, Padilla et al, 2010) sin embargo corresponde a la proporción poblacional de enfermos diabéticos (Federación Mexicana de Diabetes, 2010), (INSP, 2007), más no así a los hipertensos (INSP, 2007), (Velázquez, Monroy O et al, 2002)) resultados similares a los presentados por Singapur (Cutler, JL et al, 2010) que solo identifica el 13\% de patologías similares.

Es probable que la población no interpretara que la pandemia por influenza A (H1N1) implicara un alto riesgo de enfermar y morir, pues los rangos de tiempo para solicitar atención médica fueron muy amplios, lo que se observó sobre todo en los pacientes que fallecieron (Pérez R., de la Rosa D., Ponce de León S, 2009).

La cianosis y la disnea, fueron los aspectos clínicos al ingreso más significativos con el riesgo de muerte, manifestada esta condición porque el $63 \%$ de los fallecidos tuvieron una oximetría menor o igual a $75 \%$ de saturación de oxígeno.

La proporción de pacientes embarazadas o puérperas, que se enfermaron, con respecto a la tasa de nacimientos del 2008 (153,738) según datos del INEGI, fue de 0.5 por 10,000 nacidos; es mínima para considerarla como una relación de riesgo para la mujer con algún estado obstétrico y difiere de otros estudios realizados en Estados Unidos, donde la tasa de embarazadas infectadas por el virus de la Influenza A (H1N1) es de cuatro veces mayor a la población general (Louie JK, Acosta M, Jamieson DJ, Honein MA, California Pandemic (H1N1) Working Group, 2010).

Considerando el análisis del tiempo promedio entre el inicio de la enfermedad y el momento de solicitar atención médica; aspecto fundamental en la diferencia entre la vida y la muerte, fue la toma del medicamento antiviral. Se pudo establecer que los pacientes que lo tomaron después de los cuatro días, tuvieron mayor riesgo de morir (Caffaratti, M y Briñón, M.C. 2004). Este tiempo tuvo un promedio de casi un 60\% de tardanza (media de 7 días, rango de 3 a 18), con respecto al grupo de los no fallecidos. La letalidad en el periodo estudiado es similar al expresado por el Committee of the WHO consultation on clinical aspects of pandemiac (H1N1) 2009 influenza 2010.

En este estudio se observaron algunas limitaciones, sobre todo en las fuentes primarias. En el expediente clínico existieron múltiples cambios en los formatos de notificación, que llevó a una deficiencia en el manejo de la información; como consecuencia muy probable de no contemplar una epidemia en el programa estatal de vigilancia epidemiológica, por una aparente inexistencia histórica de influenza estacional en el estado; a pesar de ser una enfermedad de notificación obligatoria de acuerdo a la normatividad de la Subsecretaria de Prevención y Control de Enfermedades, y de la Dirección 
de Epidemiología, con base en la NOM-017-SSA2-1994, para la vigilancia epidemiológica de la Secretaria de Salud (Secretaría de Salud,1999).

En el 50\%, los expedientes no se encontró una historia clínica completa, y para obtener datos sociodemográficos y de antecedentes, fue necesario recurrir a diferentes notas tanto médicas, como de trabajo social, entre otras. Con respecto a la generalización de estos hallazgos, debemos ser cautelosos, ya que, los casos del estudio, representan una pequeña proporción de la realidad de casos que hubo durante la epidemia y que requerían hospitalización, la segunda limitación del estudio es que fue un estudio de casos hospitalizados, por lo que las características epidemiológicas, no pueden ser estimadas exactamente, al no ser un estudio basado en población general.

Dentro de las limitaciones del estudio, podemos señalar que sus hallazgos no pueden ser generalizables a una población mayor, por las siguientes razones: los casos provienen de un solo hospital, por lo que muchos otros casos pudieran no formar parte del estudio; no es un estudio basado en la comunidad, por lo que las medidas epidemiológicas obtenidas podrán tener cierto sesgo, por último la dispersión geográfica de la infección no fue tomada en cuenta, y pudiera tener un impacto importante en la morbilidad

REFERENCIAS 
Ayora -Talavera G. (1999) Influenza: Historia de una enfermedad. Mérida. Revista Biomédica, 10(1): 57-61.

Caffaratti, M y Briñón, M.C. (2004). Oseltamivir y Zanamivir. Nuevos antivirales para el tratamiento de la gripe. Córdova, España: Universidad Nacional de Córdova, 1-13.

CDC (2010). 2009 H1 N1 Flu. Disponible en https://www.cdc.gov/h1n1flu/ (consultado el 24 de febrero del 2018).

CDC (2019). Influenza (gripe). Tipos de virus de influenza. Disponible en https://espanol.cdc.gov/enes/flu/about/viruses/types.htm (consultado el 13 de enero del 2020)

Caucheme S, Donnelly CA, Carrie R, Azra C G, Christophe F, Charlotte K, Lyn F, Neil MF. (2009). Household Transmission of 2009 Pandemic Influenza A (H1N1) Virus in the United States. The New England journal of medicine, 361(27): 2619-2627.

Committee of the wHO consultation on clinical aspects of pandemic (H1N1) 2009 influenza. (2010). Clinical aspects of pandemic 2009 influenza A (H1N1) virus infection. New England Journal of Medicine, 362 (6): 1708-1719.

Cutter JL, Ang LW, Lay FY, Subramony H, Ma S, James L. (2010). Outbreak of Pandemic Influenza A (H1N1-2009) in Singapore, Acad Med Singapore, 39 (4): 273-282.

Daewood Fatimah S, Luliano A Danielle, Reed Carrie, Metzer Martin I, Shay David K, Cheng Po-Yung, Bandaraneyake Don, Breiman Robert F, Brooks W Abdullah, Buchy Phillippe, Feikin Daniel R, Fowler Karen B, Gordon Aubree, Tran Hien Nguyen, Horby Peter, Huang Q Sue, Katz Mark A, Krishand Anand, Lal Renu, Montgomery Joel M, Melbak Kare, Pebody Richard, Presanis Ann M, Razuri Hugo, Steens Anneke, Tinoko Yeni O, Wallinga jacco, Yu Hongjie, Vong Sirenda, Bresse Joseph, Widdowson Marc Alain (2012). Estimated global mortality associated with the first 12 months of the 2009 pandemic influenza A H1N1 virus circulation: a modelling study. The lancet Vol. 12. Sept. 2012. Disponible en http://dx.doi.org/10.1016/S1473-3099(12)70121-4 (consultado el 21 de febrero del 2018)

Duarte PAD, Alisson V, Nazah Chmy, De Oliveira M, Tannous L, Duarte C.,... Réa.Neto A. (2009). Outcome of influenza A (H1N1) patients admitted to intensive care units in the Paraná state, Brazil. Rev Bras Ter Intensiva. 21(3): 231-236.

Fajardo Dolci German E, Hernández Torres Francisco, Santacruz Varela Javier, Rodríguez Suárez Javier, Lami Phillippe, Arboleya Casanova Heberto, Gutiérrez Vega Rafael, Lee Gabriel Manuell, Cordova Villalobos José Angel (2009). Perfil epidemiológico de la mortalidad por influenza humana A (H1N1) en México. Salud Pública de México. 51, 361-371. 
Federación Mexicana de Diabetes, A.C. (2010). Diabetes en números. Chiapas. Recuperado de http://www.fmdiabetes.org/v2/paginas/d_numeros.php.

Instituto Nacional de Salud Pública. (2007). Encuesta Nacional de Salud y Nutrición 2006. Resultados por entidad federativa, Chiapas. 61-71.

Jain, Seema, y otros. Hospitalized Patients with 2009 H1N1 Influenza in the United States, April-June 2009. Atlanta: new england journal medicine, november 12, 2009, Vol. 361; 20, págs. 1935-1944.

Kumar A, Zarychansky R, Pinto R, Cook DJ, Marshal J, Lacroix J.,...Canadian Critical Care Trials Group H1N1 Collaborative. (2009). Critically Ill Patients With 2009 Influenza A (H1N1) Infection in Canada. Journal American Medical Association. 302 (17): 1872-1879.

Libster R, Bugna J, Coviello S, Hijano DR, Dunaiewsky M, Reynoso N.,... Polack FP. (2010). Pediatric hospitalizations associated with 2009 pandemic influenza a (H1N1) in Argentina, New England Journal of Medicine. 362 (1) $45-55$.

López I, Solís AM, López V, García Rivas D y Lozano JJ. (2010). Perfil epidemiológico de la infección por el virus de la influenza A H1N1 en un hospital de concentración de la Secretaría de salud del Distrito Federal. Medicina Interna de México. 26 (2): 123-129.

Louie JK, Acosta M, Winter K, Jean C, Gavali S, Schechter R.,...California Pandemic (H1N1) Working Group. (2009). Factors associated with death or hospitalization due to pandemic 2009 influenza A (H1N1) infection in California. The Journal off the American Medical Association, 302 (17): 1896-1902.

Louie JK, Acosta M, Jamieson DJ, Honein MA, California Pandemic (H1N1) Working Group (2010). Severe 2009 H1N1 Influenza in Pregnant and Postpartum Women in California. The New England journal of medicine. 362 (1): $27-35$.

McCloskey Brian, Dar Osman, Zumla Alimuddin y Heymann David L (2014). Emerging infectious diseases and pandemial potential: status quo and reducing risk of global spread. Disponible en www.thelancet. com/infection. Vol.14. Disponible en http://dx.doi.org/10.1016/S14733099(14)70846-1 (consultado el 02 de febrero del 2018)

Mehdi Gooya M, Sorous M, Mokhtari-Azad T, Hagdoost A, Hemati P, Moghadami M.,...Lankarani K. (2009). Influenza A (H1N1) pandemic in Iran: Report of first confirmed cases from June to November 2009. Center for Infectious Disease Control, Ministry of Health and Medical Education, Tehran, Islamic republic of Iran. Tehran. 1-17.

ops. Informe de la situación de influenza (2019). Actualización regional SE 1, 2019. Disponible en https://www.paho.org/hq/index. php?option=com_content\&view=article\&id=3352:influenza-situationreport\&Itemid=2469\&lang=es (consultado el 13 de enero del 2020). 
Organización Panamericana de la Salud. (2009). Casos de influenza por un nuevo subtipo: Actualización para las Américas. 1-2, Boletín.

O'Riordan y Sean. (2009). Risk factors and outcomes among children admitted to hospital with pandemic H1N1 influenza. Toronto. Canadian Medical Association Journal.1o (1503): 1-6. cmaj.091724.

Perez-Padilla, Rogelio; de la Rosa-Zamboni, Daniela; Ponce de Leon, Samuel (2009). Pneumonia and Respiratory Failure from Swine-origin Influenza A (H1N1) in México. [ed.] Instituto Nacional de Enfermedades Respiratorias. México: s.n., august 13, 2009, New England Journal Medicine, Vol. 361; 7, págs. 680-689.

Secretaría de Salud. NORMA OFICIAL MEXICANA NOM-017-SSA2-1994, PARA la vigilancia epidemiológica. México, México: Comité Consultivo Nacional, 19 de octubre de 1999.

Secretaría de Salud (2019). Generalidades de influenza y cierre preliminar de la temporada 2018-2019 (Segunda y última parte). Vigilancia epidemiológica semana 52, 2019. Boletín epidemiológico. Sistema Nacional de Vigilancia Epidemiológica. Sistema único de información.

Telo Velosa CS y Silva Marisela. (2009). Influenza humana a H1N1: pandemia esperada. Boletín Venezolano de Infectología. 20(1): 6-11.

The National Influenza A Pandemic (H1N1) 2009 Clinical Investigation Group of China. (2009). Clinical Features of the Initial Cases of 2009 Pandemic Influenza A (H1N1) Virus Infection in China. Beijing. The new England journal of medicine. 361(26): 2507-2517.

Velázquez O., Rosas M., Lara A., Pastelín G, Grupos Ensa 2000 y Attie F, Tapia R. (2002). Hipertensión arterial en México: resultados de la encuesta nacional de salud (ENSA) 2000. Imbiomed. 3(3): 771-785.

Virus investigation team. (2009). Emergence of a novel swine-origin Influenza $\mathrm{A}(\mathrm{H} 1 \mathrm{~N} 1)$ virus in humans. New England Journal of medicine. 360(25): 2605-2615.

Volkow Patricia, Bautista Edgar, De la Rosa Margarita, Manzano Graciela, Muñoz Torrico Marcela Veronica, Pérez Padilla Rogelio (2011). La respuesta de las unidades de terapia intensiva frente a la pandemia de influenza A (H1N1) 2009: la experiencia en Chiapas, México. Salud Pública de México. 53, 345-353.

World Health Organization. (2009). WHO ad hoc scientific teleconference on the current influenza A (H1N1) situation. 1-6. 